\title{
Editorial
}

\section{Calories do not add up}

'A calorie is a calorie' is an ambiguous phrase. In one sense it is trivially true, as in Gertrude Stein's famous phrase, 'A rose is a rose is a rose is a rose'. In another sense it is obviously questionable, because it assumes no difference between the energy produced by burning food in a bomb calorimeter and that generated by metabolic processes. It also assumes that as far as effect on body weight is concerned, fat is bad because fat by itself is calorie-dense per unit of weight, and carbohydrate and protein are good because they are relatively low in calories. It further assumes that in terms of weight gained or lost, there is no difference between diets that include the same number of kilocalories say from rice, greens and fish, or from French fries, burgers and cola, or from Danish pastries, frappuccinos and gin slings.

In their tightly argued, elegant and lucid commentary in this issue ${ }^{(1)}$, Sean Lucan of the Albert Einstein College of Medicine, New York, and James DiNicolantonio of the Mid America Heart Institute at Saint Luke's Hospital, Kansas City state that these assumptions, which have been in the mainstream of nutritional teaching and practice now for a century and more, are wrong. It is after all common sense to assume, failing consistent repeated hard evidence to the contrary, that living organisms are not machines. Also, the typical experiences of those whose body weight increases or who attempt to maintain decreased body weight - or rather, body fat - contradict the 'a calorie is a calorie' mantra.

Lucan and DiNicolantonio state that common sense and experience are now confirmed by reliable investigations. Thus:

'... a calorie's worth of salmon (largely protein) and a calorie's worth of olive oil (pure fat) have very different biological effects from a calorie's worth of white rice (refined carbohydrate) or a calorie's worth of vodka (mostly alcohol) - particularly with regard to body weight/body fatness. Indeed, scientists have recognized differences in the weightrelated physiological effects of different calorie sources for more than half a century. Although much early knowledge was based on animal studies, subsequent studies in human subjects have shown that calorie-providing proteins, fats, carbohydrates and alcohol each have substantially different effects on a variety of physiological pathways and hormones relevant to satiety, food consumption, weight maintenance and body fat composition: for example, different effects on ghrelin (an appetite-stimulating hormone), leptin (an appetite-suppressing hormone), glucagon (a hormone that raises blood sugar) and insulin (a hormone that lowers blood sugar).'

All this is not just a matter of academic interest. Obesity and diabetes - the disease most closely causally related to obesity - are now uncontrolled pandemics. The DirectorGeneral of the WHO recently pointed out that no country has succeeded in controlling them ${ }^{(2)}$. Lucan and DiNicolantonio indicate that one reason for this is that standard advice to 'control calories' is wrong, and also distracts attention from ways of life including dietary patterns most likely to prevent weight increase and to maintain a healthy weight:

"The problem with trying to "eat less" and "move more" to achieve - and more importantly, maintain caloric deficit or negative energy balance is that it is practically and biologically implausible. Practically, even the most motivated, informed and knowledgeable individuals are unlikely to be able to estimate their actual calorie intake (not just ingested, informed by misleading food labels, but absorbed) or their actual calorie expenditure (not just in physical activity but in variably efficient, silent and constantly fluctuating digestive and metabolic processes) and do so with sufficient accuracy and precision to maintain any kind of useful real-time calorie balance sheets. Biologically, calorie intake and calorie expenditure are coupled. Unless substantial uncoupling occurs, reducing calories consumed will necessarily result in a compensatory drive to reduce calories expended and vice versa. For this reason, people who try underconsuming calories become tired (an expenditure compensation) and hungry (an intake compensation)...'

This is indeed a very hot topic. Government regulatory agencies emphasise calories on the mandatory nutrition labels of processed food products and invite consumers to calculate their 'daily values' of calories in terms of 'reference' averages of energy turnover. The significance of macronutrients in the products is gauged on these labels in terms of their percentage contribution from often implausibly small stated serving sizes to calorie 'daily values'. Manufacturers love this mystifying and obviously almost useless system. It lends weight to another ambiguous phrase that valorises packaged 'convenience' products; this is of course 'there are no good or bad foods, only good or bad diets'.

An additional concern is that many influential nutrition scientists still think inside the 'a calorie is a calorie' box. 
Thus, for well over half a century, government, industry and science have converged in support of a general theory of food, nutrition, health and disease which, Lucan and DiNicolantonio come close to stating, is just plain wrong. If so, a foundation stone of modern nutrition science has crumbled.

So what to do? Agreeing that a calorie in the meter is not a calorie in the body will not of itself check and reverse rates of obesity, diabetes and other related diseases. In common with a rapidly growing number of colleagues who think out of the box, Lucan and DiNicolantonio believe that the first focus needs to be dietary constituents that are most rapidly absorbed by the body. Thus:

'Focusing quantitatively, particularly on the calories available from specific foods, fails to recognize the broader metabolic effects of foods themselves. Foods that are highly processed and comprised mostly of rapidly absorbable sugars and starches may be of greatest concern. Such carbohydrates may induce neurohormonal changes that might, in turn, help produce the overeating and inactivity often interpreted as causative for obesity. In other words, unhealthy foods may make double victims of their consumers, who may not only become obese by eating them but also receive harsh criticism for their substantial appetites and apparent laziness that result.'

Although we agree with the focus on rapidly absorbable carbohydrates, there is one important missing element in this respect. In common with colleagues from countries whose food supplies are dominated and even saturated with ultra-processed ready-to-consume products ${ }^{(3)}$, Lucan and DiNicolantonio do not consider how foods are prepared, combined and consumed, and they overlook meals. Minimally processed foods that appear 'bad' on a glycaemic index based on individual analysis, the modern analogue of a bomb calorimeter, are mostly not eaten by themselves. For example, white rice, mentioned in the commentary as an example of rapidly absorbable carbohydrates, is a staple in several countries in Asia and Latin America where it is consumed with vegetables or legumes and as part of freshly cooked meals often enjoyed mindfully in company. In this situation, its metabolic effect is quite different from that of individually analysed rice. The new food- and meal-based Dietary Guidelines issued by the Brazilian federal government ${ }^{(4)}$ incorporate consideration of meals and also the quality factors of commensality and conviviality. We invite Sean Lucan, James DiNicolantonio and others to do the same.

Carlos A. Monteiro Advisory Board, Public Health Nutrition, Department of Nutrition, School of Public Health, University of São Paulo, São Paulo, Brazil, E-mail: carlosam@usp.br.

Geoffrey Cannon Centre for Epidemiological Studies in Health and Nutrition, University of São Paulo, São Paulo, Brazil

\section{References}

1. Lucan SC \& DiNicolantonio JJ (2015) How calorie-focused thinking about obesity and related diseases may mislead and harm public health. An alternative. Public Health Nutr 18, 571-581.

2. Chan M (2013) WHO Director-General addresses health promotion conference. Opening address at the 8th Global Conference on Health Promotion, Helsinki, Finland, 10 June 2013. http://www.who.int/dg/speeches/2013/health_ promotion_20130610/en/ (accessed January 2015).

3. Monteiro CA \& Cannon G (2012) The impact of transnational 'Big Food' companies on the South: a view from Brazil. PLoS Med 9, e1001252.

4. Ministry of Health of Brazil, Secretariat of Health Care, Primary Health Care Department (2014) Dietary Guidelines for the Brazilian Population. Brasilia: Ministry of Health of Brazil; available at: http://189.28.128.100/dab/docs/portaldab/ publicacoes/guia_alimentar_populacao_ingles.pdf (accessed January 2015). 